\title{
Wer das Ziel kennt ...
}

\section{Christoph Bosshard}

Dr. med., Vizepräsident der FMH, Departementsverantwortlicher Daten, Demographie und Qualität

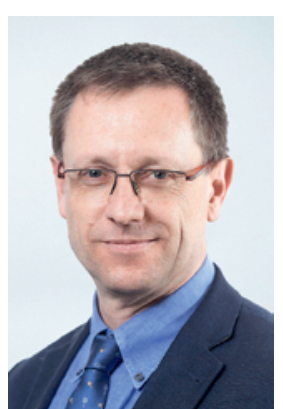

«Wer das Ziel kennt, kann entscheiden, wer entscheidet, findet Ruhe, wer Ruhe findet, ist sicher, wer sicher ist, kann überlegen, wer überlegt, kann verbessern", lehrte uns Konfuzius bereits vor 2500 Jahren. Schön und gut, aber kennen wir das Ziel? Oder: die Ziele? Sind wir uns darüber einig? Welche Wege stehen zur Verfügung? Wie steht es um die Beschaffenheit des Terrains? Verfügen wir über die notwendigen verlässlichen Navigationsinstrumente? Eine ganze Reihe von Fragen stehen im Raum, welche der Beantwortung harren. Viele dieser Fragen haben wir im Rahmen unserer Dialoggruppe Versorgungsforschung zusammen mit Prof. Dr. Marcel Zwahlen, Institut für Sozial- und Präventivmedizin (ISPM) der Universität Bern, besprochen. Obschon diese Fragen unbestritten eine zentrale Bedeutung haben, fehlen bei etwa zwei Dritteln sowohl die notwendigen Daten wie auch die taugliche Methodik zur Beantwortung. Es ist ja nicht so, dass wir in Datenarmut leben würden. Zum Teil mangelt es jedoch an der für die Fragestellung geeigneten Primärdaten-Qualität sowie auch an der Datenstruktur. Des Weiteren müssen wir uns bezüglich Methodik noch weiterentwickeln. Der Bundesrat hat seine gesundheitspolitische Strategie 2020-2030 in die Vernehmlassung geschickt. Darin enthalten sind klare

\section{Alleinige Korrelationsbilder sind keine ausreichende Grundlage für eine Kausalitäts- diskussion.}

Bedürfnisse und auch Forderungen bezüglich Digitalisierung und Datennutzung. Wir als Akteure des Schweizer Gesundheitswesens müssen hier zusammenarbeiten und das Fachwissen der FMH-Organisationen einfliessen lassen. Ansonsten riskieren wir, dass auf allen Ebenen Fehler in Kauf genommen werden und sich dann fortpflanzen, begonnen bei der Primärdaten-Qualität über die Datenstruktur, die Variablen- bildung bis hin zur Auswertung und Interpretation. Als kleines Beispiel sei hier folgende Studie angefügt, deren Resultat zu einer gewissen Konsternation geführt hat: Die Studie untersuchte die Mortalität bei akutem Koronarsyndrom in Abhängigkeit zur Distanz zur nächsten Notfallstation. Eine unerwartet schwache bis keine Korrelation wurde festgestellt. Was jetzt? Brauchen wir somit keine Notfallstationen mehr? Kann man geradeso gut oder noch besser zu Hause sterben? Oder hat das Problem allenfalls bereits bei der Fragestellung angefangen? symptoms-to-balloon time, heisst das Stich-

Das Ziel ist nicht, viele Fragen mit vielen Daten zu beantworten, sondern die richtigen Fragen im richtigen Kontext zu stellen.

wort! Nicht die Nähe zur nächsten Notfallstation, sondern die Dauer bis zur Versorgung in einem Herzkatheterlabor wäre wohl die sinnbildendere Frage gewesen. Alleinige Korrelationsbilder sind keine ausreichende Grundlage für eine Kausalitätsdiskussion. Es geht also nicht darum, möglichst viele Fragen mit möglichst vielen Daten zu beantworten. Vielmehr sollten die richtigen Fragen im richtigen Kontext mit den dafür richtigen Daten und Variablen bearbeitet werden.

Auch im medizinischen Alltag setzt sich die Ärzteschaft dafür ein, das Richtige richtig zu tun. So gilt es auch in anderen Bereichen, nicht das Falsche richtig zu tun. Fehler zu Beginn dieses Prozesses sind fast nicht mehr zu korrigieren. Am Ende geht es auch um Interpretationen, Schlussfolgerungen und Entscheide. Die dafür gemäss Gesetz zuständigen Instanzen brauchen hier die breite Zusammenarbeit und Unterstützung auch unserer Organisationen. Die FMH und die Dialoggruppe Versorgungsforschung werden sich voller Engagement dafür einsetzen, hier einen wichtigen Beitrag zu leisten. Also: Ein Ziel haben wir ja schon! Packen wir es gemeinsam an! 